\title{
Short pulse generation and high power emission of Quantum Cascade lasers
}

\author{
D. Bachmann 1 , M. Rösch³ , M. Kainz ${ }^{1}$, S. Schönhuber ${ }^{1}$, M. Brandstetter ${ }^{1}$, G. Scalari³ ${ }^{3}$ M. Beck ${ }^{3}$, J. \\ Faist $^{3}$, M.A. Andrews ${ }^{2}$, G. Strasser ${ }^{2}$, J. Darmo ${ }^{1}$, K. Unterrainer ${ }^{1}$ \\ ${ }^{1}$ Photonics Institute and Center for Micro\&Nanostructures, TU Wien, A-1040 Vienna, Austria \\ ${ }^{2}$ Institute for Solid State Electronics and Center for Micro\&Nanostructures, TU Wien, A-1040 Vienna, Austria \\ ${ }^{3}$ Institute for Quantum Electronics, ETH Zürich, CH-8093 Zürich, Switzerland
}

\begin{abstract}
A unique feature of Quantum Cascade lasers is the possibility to stack active regions. Using a three stack heterogeneous Quantum Cascade laser ultra broadband amplification and short pulse generation is demonstrated. High power output is achieved by two stacked symmetric active regions.
\end{abstract}

\section{INTRODUCTION}

$\mathrm{Q}$ uantum cascade lasers (QCLs) are most successful quantum devices which cover a broad spectral range from the infrared to the THz. However, their operation is still limited in terms of operating temperature, output power and spectral bandwidth.

A unique feature of QCLs is that active regions with different transition energies can be stacked. This allows the realization of broadband lasers. We employ such a broadband Quantum Cascade structure as amplifier for few-cycle $\mathrm{THz}$ pulses generated by femtosecond lasers. Another strategy to improve the performance is to increase the number of cascades in the active region. We present a direct wafer-bonding technique to increase the thickness of the active region by stacking two active regions.

\section{BROADBAND AMPLIFICATION}

THz QCLs with broadband gain are highly desirable for many applications ranging from $\mathrm{THz}$ amplifiers for timedomain spectroscopy to tunable external-cavity lasers. It has been shown that $\mathrm{THz}$ QCLs can be actively mode-locked and that they can form a frequency comb. All these results were limited by a rather narrow gain bandwidth. A very promising approach to increase the bandwidth of QCLs is to stack different active regions with individually designed emission frequencies into a single waveguide. This concept of heterogeneous active regions was initially applied to midinfrared QCLs and later adapted to $\mathrm{THz}$ QCLs, where broadband lasing with bandwidths up to $\sim 1 \mathrm{THz}$ has been demonstrated [1].

Such heterogeneous active regions offer high design freedom to shape the optical gain by changing the strength and spectral separation of the individual optical transitions, as well as the number of cascades and their composition in the waveguide. Because additional requirements, such as identical threshold currents of the different stacks, make the overall design very crucial, THz-time domain spectroscopy gain measurements provide valuable information for the bandstructure design and consequently enable further improvement of heterogeneous active regions. A detailed characterization of an ultra- broadband multi-stack THz QCL gain medium, confined in a metal-metal waveguide is performed. The near unity confinement of the optical mode, and the almost homogeneous field distribution across the entire active region, makes the metal-metal waveguide highly suitable for heterogeneous $\mathrm{THz}$ QCLs. In order to overcome the issue of the very low coupling efficiency of the broadband $\mathrm{THz}$ pulses into the subwavelength metal-metal waveguide, we employ a coupled cavity geometry.

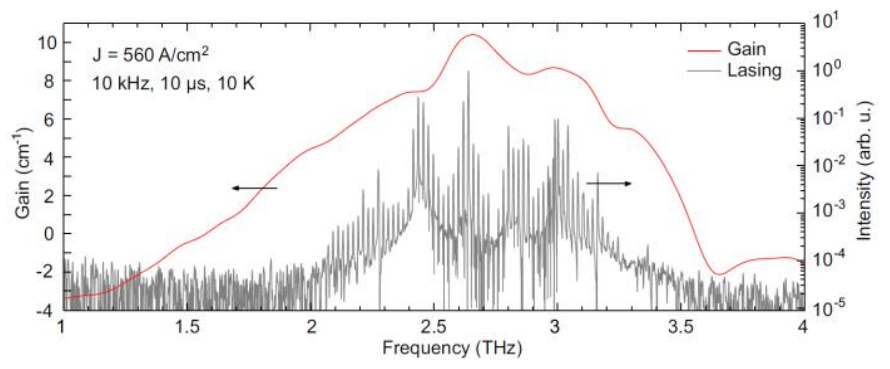

Figure 1: Comparison of the intensity gain, measured by $\mathrm{THz}$ timedomain spectroscopy in a coupled cavity configuration, and the lasing spectrum (FTIR) of an ultra-broadband THz QCL.

The spectral gain of a multi-stack terahertz quantum cascade laser, composed of three individually designed active regions, is studied as a function of bias and temperature using terahertz time-domain spectroscopy. The gain associated with the particular quantum cascade structures clamps at different driving currents and saturates to different values. The heterogeneous active region exhibits a spectral gain with full width at half-maximum of $1.1 \mathrm{THz}$. Bandwidth and spectral position of the measured gain match with the broadband laser emission [2]. In addition, we show that lateral mode control is essential for the suppression of higher order modes. Injection seeding of these devices resulted in generation of 2.5 ps short THz pulses [3].

\section{HIGH OUTPUT POWER}

One approach to increase the optical gain in a terahertz QCL device is to replace the standard GaAs/AlGaAs material system with InGaAs/GaAsSb or InGaAs/InAlAs due to the lower effective electron mass [4]. Another strategy is to simply increase the number of cascades in the active region. However, long-run stability and growth time of molecular beam epitaxy basically limits the maximum active region thickness to around 10-15 $\mu \mathrm{m}$. To circumvent this growth 
problem we developed a direct wafer-bonding technique to double the thickness of the active region by stacking the same active region. This method can be readily applied to standard designs and double metal waveguides due to the flip-chip fabrication. Surface-plasmon waveguides on the other hand exhibit lower waveguide losses and better outcoupling properties, which are perfect for high power devices. Thicker active regions are especially suited for surface-plasmon waveguides as the confinement factor is increased. However, in order to realize such wafer-bonded surface-plasmon terahertz QCLs symmetric, bidirectionally working active regions are required because the two individual substacks are biased in opposite directions with respect to the growth direction.

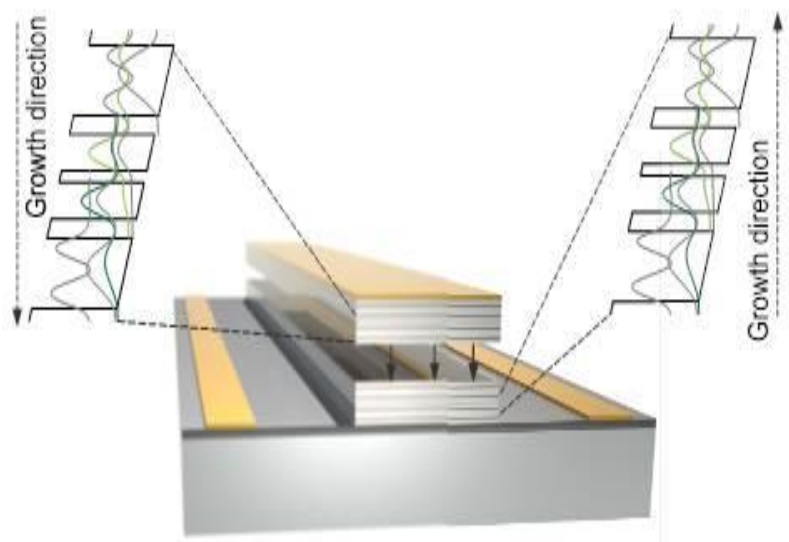

Figure 2: Waferbonding of two symmetric Quantum Cascade active regions

Due to their operation principle QCLs allow the design of symmetric devices. We have developed symmetric band structure designs based on a three-well phonon depletion scheme.

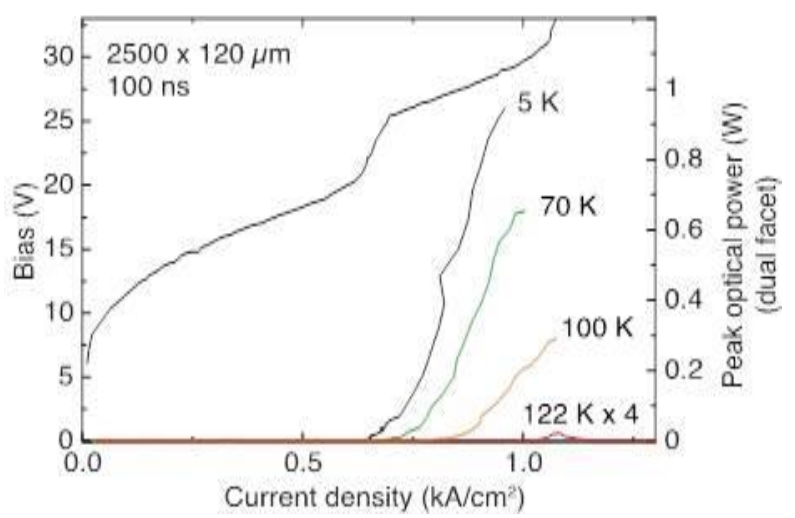

Figure 3: Intensity (voltage) versus current characteristics of the wafer-bonded device

Fig. 2 illustrates a surface-plasmon wafer-bonded terahertz QCL consisting of two symmetric active regions where the upper part is flipped upside down during fabrication. Consequently the electron transport occurs in and against the growth direction for the respective active region part. The resulting thicker waveguide geometry $(20 \mu \mathrm{m}$ instead of 10 $\mu \mathrm{m})$ yields a significant advantage: The confinement factor $\Gamma$ is increased from 0.288 to 0.472 while the waveguide losses remain constant. The light-current-voltage (LIV) characteristics of the wafer-bonded device are shown in Fig. 3. The peak optical output power from the two facets reaches $0.94 \mathrm{~W}$ at $5 \mathrm{~K}$ and still more than $0.6 \mathrm{~W}$ at $70 \mathrm{~K}$ [5].

\section{REFERENCES}

[1]. M. Rösch, G. Scalari, M. Beck, and J. Faist, “Octave spanning semiconductor laser," Nat. Photonics 9, 42-47, (2015).

[2]. D. Bachmann et.al, "Broadband terahertz amplification in a heterogeneous quantum cascade laser", Opt. Express 23, 3117 (2015)

[3]. D. Bachmann, M. Rösch, M. Süss, M. Beck, K. Unterrainer, J. Darmo, J. Faist, G. Scalari, "Short pulse generation and mode control of broadband terahertz quantum cascade lasers", Optica, 3, 1087 (2016)

[4]. C. Deutsch et al., "High performance InGaAs/GaAsSb terahertz quantum cascade lasers operating up to 142 K", Applied Physics Letters 101, 211117 (2012).

[5]. M. Brandstetter et al., "High power terahertz quantum cascade lasers with symmetric wafer bonded active regions", Applied Physics Letters 103, 171113 (2013).

(c) (c) 2017 IEEE. Personal use of this material is permitted. Permission from IEEE must be obtained for all other uses, in any current or future media, including reprinting/republishing this material for advertising or promotional purposes, creating new collective works, for resale or redistribution to servers or lists, or reuse of any copyrighted component of this work in other works.

This is the accepted version of D. Bachmann et al., "Short pulse generation and high power emission of Quantum Cascade lasers," 2017 42rd International Conference on Infrared, Millimeter, and Terahertz Waves (IRMMW-THz), Cancun, Mexico, 2017, doi: 10.1109/IRMMW-THz.2017.8067124 